\title{
WEAK EMISSION-LINE QUASARS IN THE CONTEXT OF A MODIFIED BALDWIN EFFECT
}

\author{
OHad Shemmer and Sara Lieber \\ Department of Physics, University of North Texas, Denton, TX 76203, USA; ohad@unt.edu \\ Received 2015 January 26; accepted 2015 March 17; published 2015 May 28
}

\begin{abstract}
We investigate the relationship between the rest-frame equivalent width (EW) of the $\mathrm{C}_{\text {IV }} \lambda 1549$ broad-emission line, monochromatic luminosity at rest-frame $5100 \AA$, and the $\mathrm{H} \beta$-based Eddington ratio in a sample of 99 ordinary quasars across the widest possible ranges of redshift $(0<z<3.5)$ and bolometric luminosity $\left(10^{44} \lesssim L \lesssim 10^{48} \mathrm{erg} \mathrm{s}^{-1}\right)$. We find that $\mathrm{EW}(\mathrm{C}$ IV $)$ is primarily anti-correlated with the Eddington ratio, a relation we refer to as a modified Baldwin effect (MBE), an extension of the result previously obtained for quasars at $z<0.5$. Based on the MBE, weak emission line quasars (WLQs), typically showing $\mathrm{EW}(\mathrm{C}$ Iv $) \lesssim 10 \AA$, are expected to have extremely high Eddington ratios. By selecting all WLQs with archival $\mathrm{H} \beta$ and $\mathrm{C}$ IV spectroscopic data, nine sources in total, we find that their $\mathrm{H} \beta$-based Eddington ratios are typical of ordinary quasars with similar redshifts and luminosities. Four of these WLQs can be accommodated by the MBE, but the other five deviate significantly from this relation, at the $\gtrsim 3 \sigma$ level, by exhibiting $\mathrm{C}$ Iv lines much weaker than predicted from their $\mathrm{H} \beta$-based Eddington ratios. Assuming the supermassive black hole masses in all quasars can be determined reliably using the single-epoch $\mathrm{H} \beta$-method, our results indicate that $\mathrm{EW}(\mathrm{C}$ Iv $)$ cannot depend solely on the Eddington ratio. We briefly discuss a strategy for further investigation into the roles that basic physical properties play in controlling the relative strengths of broad-emission lines in quasars.
\end{abstract}

Key words: galaxies: active - galaxies: nuclei - quasars: emission lines - quasars: general

\section{INTRODUCTION}

The classical "Baldwin effect" is an anti-correlation between the rest-frame equivalent width $(\mathrm{EW})$ of a broad-emission line region (BELR) line and quasar luminosity, first observed for the C IV $\lambda 1549$ line (Baldwin 1977). This anti-correlation is stronger and steeper for BELR lines with higher ionization potentials ( $\chi_{\text {ion }}$; Dietrich et al. 2002), but it involves substantial scatter, hampering its use as a cosmological probe (Osmer \& Shields 1999). Considerable effort has been invested in attempts to minimize this scatter, using partial-correlation and principal-component analyses involving emission-line as well as broad-band spectroscopic data (e.g., Wilkes et al. 1999; Shang et al. 2003), but the exact cause of the Baldwin effect remains elusive. A dependence on the shape of the continuumsource spectral energy distribution (SED; Zheng \& Malkan 1993), cosmic evolution (Green et al. 2001), or the supermassive black hole mass $\left(M_{\mathrm{BH}}\right.$; Xu et al. 2008) being the primary physical driver for the EW-luminosity anti-correlation are among the explanations proposed for this effect. It had also been speculated that the Baldwin effect depends largely on the normalized accretion rate, in terms of the Eddington ratio, $L / L_{\text {Edd }}$, where $L$ is the bolometric luminosity and $L_{\text {Edd }}$ is the Eddington luminosity (e.g., Brotherton \& Francis 1999; Wills et al. 1999; Bachev et al. 2004).

Using the empirical BELR size-luminosity relation (Kaspi et al. 2005; Bentz et al. 2009) and assuming the BELR gas is virialized, $M_{\mathrm{BH}}$ takes a general expression of the form $M_{\mathrm{BH}} \propto\left(\nu L_{\nu}\right)^{0.5} \mathrm{FWHM}^{2}$; the Eddington ratio can therefore be expressed as $L / L_{\mathrm{Edd}} \propto\left(\nu L_{\nu}\right)^{0.5} \mathrm{FWHM}^{-2}$, where $\nu L_{\nu}$ and FWHM typically correspond to the monochromatic luminosity at rest-frame $5100 \AA$ and the full width at half maximum intensity of the broad $\mathrm{H} \beta^{1}$ line, respectively (see also, e.g., Laor 1998).

\footnotetext{
Attempts to determine $M_{\mathrm{BH}}$ from high-ionization BELR lines, such as C IV, may yield unreliable results, since the line profiles are complicated by a nonvirial (i.e., "wind") component (e.g., Baskin \& Laor 2005; Richards et al. 2011; Shen \& Liu 2012; Trakhtenbrot \& Netzer 2012).
}

Utilizing optical spectroscopic data for a sample of 81 quasars with $L \sim 10^{44}-10^{46} \mathrm{erg} \mathrm{s}^{-1}$ at $z<0.5$ from Boroson \& Green (1992), and for which high-quality archival UV spectroscopic data were available, Baskin \& Laor (2004, hereafter BL04) found a significant anti-correlation between $\mathrm{EW}(\mathrm{C}$ Iv $)$ and $\mathrm{H} \beta$-based $L / L_{\mathrm{Edd}}$; they did not find a significant correlation between $\mathrm{EW}(\mathrm{C}$ IV $)$ and monochromatic luminosity at rest-frame $3000 \AA$. BL04 argued that most of the scatter in the classical Baldwin effect is produced by a range of $L / L_{\text {Edd }}$ at a given $L$, driven by a range in $\operatorname{FWHM}(\mathrm{H} \beta)$. This scatter is minimized considerably when a combination of $\operatorname{FWHM}(\mathrm{H} \beta)$ and luminosity, i.e., the Eddington ratio, is employed, thus strengthening the anti-correlation with $\mathrm{EW}\left(\mathrm{C}_{\mathrm{IV}}\right)$. BL04 claimed that the classical Baldwin effect is only a secondary effect since, typically, more luminous quasars also have higher Eddington ratios. Dong et al. (2009) report a similar result for the EW of the Mg II $\lambda \lambda 2796,2803$ doublet using Mg II-based $L / L_{\text {Edd }}$ determinations for a sample of 2092 active galactic nuclei at $0.45 \leqslant z \leqslant 0.8$, suggesting that the Baldwin effect is governed by $L / L_{\mathrm{Edd}}$.

In this work, we extend the BL04 analysis by including quasars with $L \sim 10^{46}-10^{48} \mathrm{erg} \mathrm{s}^{-1}$ at $2<z<3.5$ that have $\mathrm{H} \beta$ spectral information from near-infrared (NIR) spectroscopy as well as $\mathrm{C}$ IV information from optical spectroscopy in order to test whether the $\mathrm{EW}(\mathrm{C}$ IV $)-L / L_{\text {Edd }}$ anti-correlation, hereafter the modified Baldwin effect (MBE), remains strong across the widest possible ranges of redshift, luminosity, and $L / L_{\text {Edd. }}$ In particular, the extension of this relationship to higher redshifts and luminosities is required in order to test the hypothesis that the extreme weakness of the $\mathrm{C}$ Iv lines in weak emission line quasars (WLQs), that typically have $\mathrm{EW}(\mathrm{C}$ IV $) \lesssim 10 \AA$ (e.g., Fan et al. 1999; Diamond-Stanic et al. 2009), is due to extremely high accretion rates in these sources (see, e.g., Leighly et al. 2007a; Shemmer et al. 2009, 2010). In Section 2 we describe the properties and spectroscopic measurements of our quasar sample, including WLQs, and in Section 3 we present the results of a correlation analysis involving $\mathrm{EW}\left(\mathrm{C}_{\mathrm{IV}}\right)$, 
monochromatic luminosity, and $L / L_{\text {Edd }}$. In Section 4 we discuss the implications of our results for quasars in general and for WLQs in particular, and in Section 5 we summarize our main conclusions. Throughout this paper, wavelengths, frequencies, and EWs are given in the rest-frame of each source. Complete source names are given in tables and figures and abbreviated names are given throughout the text. Luminosity distances were computed using the standard cosmological model $\left(\Omega_{\Lambda}=0.7, \Omega_{\mathrm{M}}=0.3\right.$, and $H_{0}=70 \quad \mathrm{~km} \mathrm{~s}^{-1} \mathrm{Mpc}^{-1}$; e.g., Spergel et al. 2007).

\section{SAMPLE SELECTION AND DATA ANALYSIS}

Our high-redshift quasar sample is drawn from the Shemmer et al. (2004, hereafter S04) and Netzer et al. (2007, hereafter N07) studies involving high-quality NIR spectra of 29 and 15 sources, respectively, covering the $\mathrm{H} \beta$ spectral region in the $2<z<3.5$ range. We exclude six radioloud quasars (RLQs) from S04, ${ }^{2}$ [HB89] 0123+257, [HB89] 0504+030, [HB89] 2126-158, TON 618, UM 632, and [HB89] 2254+024, as sources having radio-loudness values of $R>100$ (where $R$ is the ratio between the flux densities at $5 \mathrm{GHz}$ and $4400 \AA$; Kellermann et al. 1989), based on the NRAO VLA Sky Survey (Condon et al. 1998) for the first three of these sources and the Faint Images of the Radio Sky at Twenty Centimeters (FIRST) survey (Becker et al. 1995) for the latter three. We also exclude [HB89] 1246-057 (from S04) and SDSS J2103-0600 (from N07) as broad absorption line (BAL) quasars, based on Osmer \& Smith (1977) and Gibson et al. (2009), respectively. The exclusion of RLQs and BAL quasars is intended to minimize potential effects of continuum boosting (see below; e.g., Meusinger \& Balafkan 2014) and absorption biases (e.g., BL04), respectively, that may result in systematic underestimations of EW(C IV).

Relevant properties of our final sample of 36 "ordinary" quasars (i.e., type 1 quasars that are not radio loud and that do not have BALs) at high redshift, hereafter the HIZ sample, are given in Table 1. We also note in Table 1 that nine of the HIZ sources are identified as BAL quasars in Trump et al. (2006) but not in the more recent BAL quasar catalog of Gibson et al. (2009); we consider these sources as non-BAL quasars and they are retained in our sample. For each source in the HIZ sample, we obtain the systemic redshift $\left(z_{\text {sys }}\right), \nu L_{\nu}(5100 \AA)$, and best-fit $\mathrm{FWHM}(\mathrm{H} \beta)$ values from Tables 1 and 2 of S04 and from Table 2 of N07. We derive the $L / L_{\text {Edd }}$ value for each source following Equation (2) of Shemmer et al. (2010),

$$
L / L_{\mathrm{Edd}}=0.13 f(L)\left[\frac{\nu L_{\nu}(5100 \AA)}{10^{44} \mathrm{erg} \mathrm{s}^{-1}}\right]^{0.5}\left[\frac{\operatorname{FWHM}(\mathrm{H} \beta)}{10^{3} \mathrm{~km} \mathrm{~s}^{-1}}\right]^{-2} \text {, }
$$

and using Equation (21) of Marconi et al. (2004) to compute $f(L)$, the luminosity-dependent bolometric correction to $\nu L_{\nu}(5100 \AA)$, which is in the range $5.42<f(L)<6.43$ for our sources.

Thirty of the HIZ sources have rest-frame UV spectra in electronic form that are publicly available; 23 spectra have been obtained from the Sloan Digital Sky Survey (SDSS; York et al. 2000) and 7 spectra have been obtained from the Two-

\footnotetext{
2 S04 have, erroneously, identified [HB89] 2132+014 as an RLQ, instead of [HB89] 2126-158. They also identified [HB89] 0329-385 and UM 645 as RLQs; however, as we mention below, these two sources have $10<R<100$ and are thus considered radio-intermediate quasars.
}

degree Field quasar redshift survey (2QZ; Croom et al. 2004); the spectral response of each 2QZ spectrum has been determined as described in S04. For each spectrum, we fitted the $\sim 1450-1700 \AA$ spectral region around the $\mathrm{C}$ IV line using a linear continuum and two Gaussian profiles, describing the entire profile of the $\mathrm{C}$ IV line. The two Gaussian profiles are used for least-squares fitting purposes only and thus are not intended to represent two physically distinct emission regions. The linear continuum was determined based on average flux densities obtained in $10 \AA$ wide intervals centered on $\lambda_{1} \simeq 1445 \AA$ and $\lambda_{2} \simeq 1695 \AA$. The $\mathrm{EW}$ of the $\mathrm{C}$ IV line in each source has been computed using the sum of the fluxes in each best-fit Gaussian profile and the best-fit linear continuum underlying the emission line. The errors on $\mathrm{EW}(\mathrm{C} \mathrm{IV})$ were estimated by repeating the fitting procedure but, for each spectrum, the two steepest continua were considered, based on the $1 \sigma$ value of the flux density in each of the two continuum intervals, i.e., fitting between $\left(f_{\lambda_{1}}+\Delta f_{\lambda_{1}}, f_{\lambda_{2}}-\Delta f_{\lambda_{2}}\right)$ and $\left(f_{\lambda_{1}}-\Delta f_{\lambda_{1}}, f_{\lambda_{2}}+\Delta f_{\lambda_{2}}\right)$.

For the six HIZ sources that lack publicly available spectra, we obtained the $\mathrm{EW}(\mathrm{C}$ IV $)$ values from the literature. The $\mathrm{EW}(\mathrm{C}$ IV $)$ values for all of the HIZ sources are given in Table 1. For the 23 sources from our HIZ sample that have SDSS spectra and for which we have measured $\mathrm{EW}(\mathrm{C}$ IV $)$ values, such values (not shown in Table 1) can also be obtained from the spectral measurements of Shen et al. (2011). For 17 of these sources, the EW(C IV) values from Shen et al. (2011) agree with our measurements, within the errors. The spectra of six sources for which the discrepancies between our measurements and the Shen et al. (2011) values are $~ 20 \%-80 \%$ have, on average, lower signal-to-noise ratios than the spectra of the 17 sources in which such discrepancies are $\lesssim 20 \%$. Replacing our EW(C IV $)$ measurements with the corresponding Shen et al. (2011) values for these six sources does not alter significantly any of our subsequent results.

We complement the HIZ sample with a subset of 63 ordinary quasars from BL04, following the exclusion of five BAL quasars, PG 0043+039, PG 2112+059 (Jannuzi et al. 1998), PG 1001+054 (Brandt et al. 2000), PG 1411+442 (Malkan et al. 1987), and PG 1416-129 (Turnshek \& Grillmair 1986), as well as 13 RLQs (with $R>100$ ), PG 0003+158, PG 0007+106, PG 1048-090, PG 1100+772, PG 1103-006, PG 1226+023, PG 1302-102, PG 1512+370, PG 1545+210, PG 1704+608, PG 2209+184, PG 2251+113, and PG 2308+098 (Boroson \& Green 1992). For each of the 63 BL04 sources, we obtain the redshift and $\operatorname{FWHM}(\mathrm{H} \beta)$ information from Tables 1 and 2 of Boroson \& Green (1992), respectively, and EW(C IV) values are obtained from Table 1 of BL04. The $\nu L_{\nu}(3000 \AA)$ values for the BL04 sources, given in Table 1 of BL04, are converted to $\nu L_{\nu}(5100 \AA)$ values, assuming an optical continuum of the form $f_{\nu} \propto \nu^{-0.5}$ (e.g., Vanden Berk et al. 2001) and correcting the luminosity distances based on our adopted cosmological parameters (see Section 1). The Eddington ratios of the BL04 sources are determined using Equation (1).

In order to test the hypothesis that WLQs are quasars with extremely high Eddington ratios (e.g., Shemmer et al. 2009, 2010), we select all the WLQs for which accurate $\mathrm{H} \beta$ properties (such as FWHM and EW) are available from the literature. For the purpose of this work, we consider all optically selected type 1 quasars that (i) have radio-loudness values of $R<100$, (ii) do not show BAL troughs in their restframe UV spectra, and (iii) have $\mathrm{EW}(\mathrm{C}$ IV $)<10 \AA$ as WLQs. 
Table 1

Basic Properties of the HIZ Sample

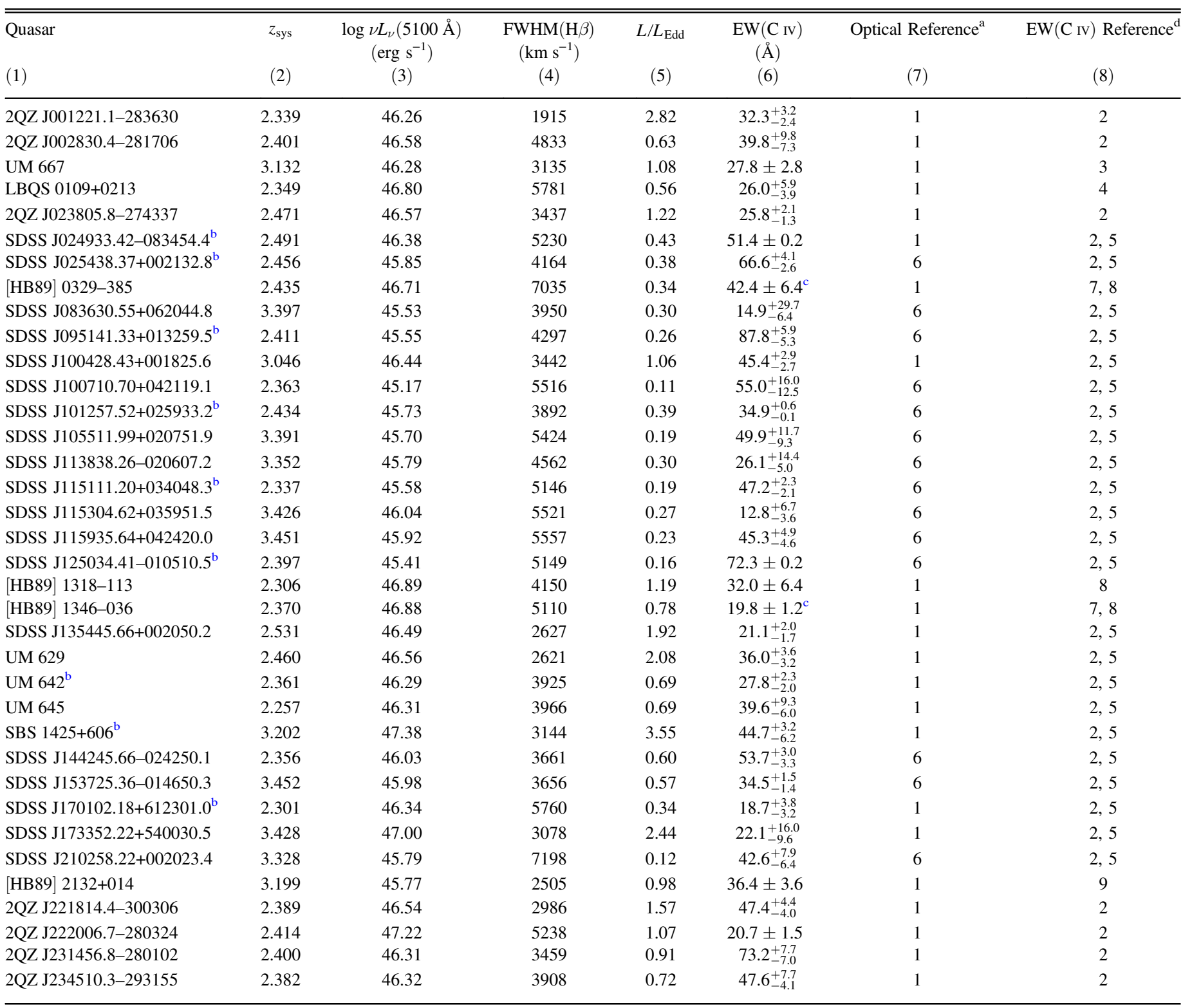

Notes.

${ }^{a}$ Source of rest-frame optical data, including $z_{\text {sys }}, \nu L_{\nu}(5100 \AA)$, and $\operatorname{FWHM}(\mathrm{H} \beta)$.

${ }^{\mathrm{b}}$ Identified as a BAL quasar in Trump et al. (2006) but not in Gibson et al. (2009).

c The EW(C IV ) value is the average of the two values given in the references; error bar is taken as one half the difference between the two values.

${ }^{\mathrm{d}}$ Unless stated otherwise, the EW(C IV) value adopted for analysis in this work is obtained from the first reference for each source.

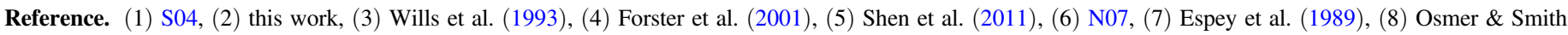
(1977), (9) Schneider et al. (1991).

The third criterion follows from the fact that $\sim 10 \AA$ marks the $3 \sigma$ threshold on the low end of lognormal fits to distributions of $\mathrm{EW}(\mathrm{C}$ IV $)$ values for quasars at $1.5 \lesssim z \lesssim 5.0$; i.e., $\lesssim 0.15 \%$ of quasars at this redshift range have $\mathrm{EW}\left(\mathrm{C}_{\mathrm{IV}}\right)<10 \AA$ (e.g., Diamond-Stanic et al. 2009; Wu et al. 2011, 2012; Plotkin et al. 2015). ${ }^{3}$ We caution that these selection criteria likely

\footnotetext{
3 There is tentative evidence that the fraction of WLQs is considerably larger than $0.15 \%$ of the entire quasar population at $z \gtrsim 5$ (see, e.g., Fan et al. 2006; Bañados et al. 2014).
}

result in a heterogenous group of quasars, and we do not expect, a priori, a common origin for the weakness of the $\mathrm{C}$ IV BELR line in all such sources. Our WLQ sample of nine sources includes SDSS J0836+1425, SDSS J1411+1402, SDSS J1417+0733, SDSS J1447-0203 (Plotkin et al. 2010, 2015), SDSS J0945+1009 (Hryniewicz et al. 2010; Plotkin et al. 2015), SDSS J1141+0219, SDSS J1237+6301 (Diamond-Stanic et al. 2009; Shemmer et al. 2010), SDSS J1521+5202 (Just et al. 2007; Wu et al. 2011), and PHL 1811 (Leighly et al. 2007a, 2007b). Table 2 
Table 2

Basic Properties of the WLQ Sample

\begin{tabular}{|c|c|c|c|c|c|c|c|}
\hline $\begin{array}{l}\text { Quasar } \\
\text { (1) } \\
\end{array}$ & $\begin{array}{l}z_{\text {sys }} \\
\text { (2) }\end{array}$ & $\begin{array}{c}\log \nu L_{\nu}(5100 \AA) \\
\left(\mathrm{erg} \mathrm{s}^{-1}\right) \\
(3)\end{array}$ & $\begin{array}{c}\operatorname{FWHM}(\mathrm{H} \beta) \\
\left(\mathrm{km} \mathrm{s}^{-1}\right) \\
(4)\end{array}$ & $\begin{array}{c}L / L_{\text {Edd }} \\
(5) \\
\end{array}$ & $\begin{array}{c}\text { EW(C IV }) \\
(\AA) \\
(6) \\
\end{array}$ & $\begin{array}{c}\text { Optical Reference }^{\mathrm{a}} \\
\text { (7) }\end{array}$ & $\begin{array}{c}\text { EW }(\mathrm{C} \text { Iv }) \text { Reference }^{\mathrm{b}} \\
(8) \\
\end{array}$ \\
\hline SDSS J094533.98+100950.1 & 1.683 & 46.17 & 4278 & 0.51 & $2.9_{-0.6}^{+0.3}$ & 1 & 1,2 \\
\hline SDSS J114153.34+021924.3 & 3.55 & 46.55 & 5900 & 0.41 & $0.4 \pm 0.2$ & 3 & 4 \\
\hline SDSS J123743.08+630144.9 & 3.49 & 46.35 & 5200 & 0.42 & $7.7 \pm 1.1$ & 3 & 4,2 \\
\hline SDSS J144741.76-020339.1 & 1.430 & 45.56 & 1923 & 1.33 & $7.7_{-1.3}^{+0.2}$ & 1 & 1 \\
\hline SDSS J152156.48+520238.5 & 2.238 & 47.14 & 5750 & 0.81 & $9.1 \pm 0.6$ & 5 & 5,2 \\
\hline PHL 1811 & 0.192 & 45.56 & 1943 & 1.30 & 6.6 & 6 & 6 \\
\hline
\end{tabular}

Notes.

${ }^{a}$ Source of rest-frame optical data, including $z_{\text {sys }}, \nu L_{\nu}(5100 \AA)$, and $\operatorname{FWHM}(\mathrm{H} \beta)$.

${ }^{b}$ The $\mathrm{EW}(\mathrm{C}$ IV $)$ value adopted for analysis in this work is obtained from the first reference for each source.

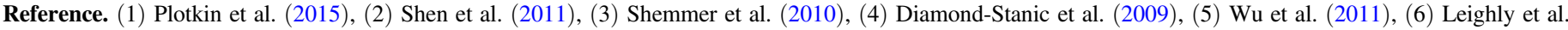
(2007a).

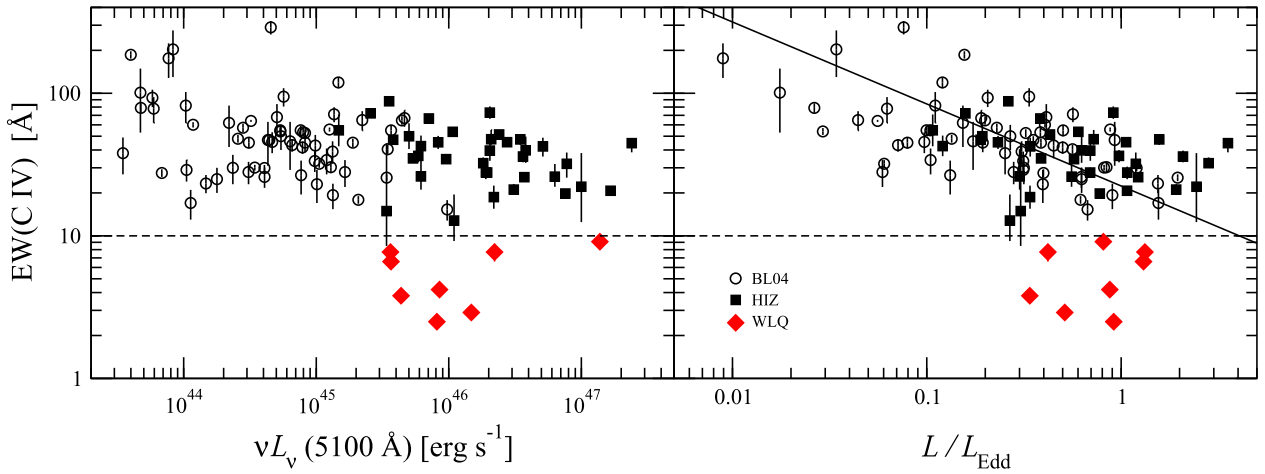

Figure 1. EW(C Iv) vs. monochromatic luminosity at $5100 \AA$ (left) and $L / L$ Edd (right). Circles, squares, and diamonds represent the BL04, HIZ, and WLQ samples, respectively. The dashed line in each panel marks the $\mathrm{EW}(\mathrm{C}$ IV $)=10 \AA$ threshold for WLQs, and the solid line in the right panel marks the BCES Bisector best-fit $\log [\mathrm{EW}(\mathrm{C}$ IV $)]-\log \left(L / L_{\text {Edd }}\right)$ relation for a combination of the BL04 and HIZ samples. The WLQ SDSS J114153.34+021924.3 with $L / L_{\mathrm{Edd}}=0.41$ and $\mathrm{EW}(\mathrm{C}$ Iv $)=0.4 \pm 0.2$ is not plotted, for clarity.

presents the $z_{\text {sys }}, \nu L_{\nu}(5100 \AA), \operatorname{FWHM}(\mathrm{H} \beta), L / L_{\text {Edd }}($ determined using Equation (1)), and $\mathrm{EW}(\mathrm{C}$ iv) values for our WLQ sample. For five SDSS sources from the WLQ sample, the $\mathrm{EW}(\mathrm{C}$ Iv) values from either Diamond-Stanic et al. (2009) or Plotkin et al. (2015) are consistent, within the errors, with the values obtained from Shen et al. (2011); Shen et al. (2011) do not provide EW(C IV) measurements for SDSS J1141+0219 and SDSS J1447-0203. For SDSS J1521+5202, Shen et al. (2011) give $\operatorname{EW}\left(C_{\text {IV }}\right)=3.0 \pm 0.2$, which is a factor of $\simeq 3$ smaller than the value reported in $\mathrm{Wu}$ et al. (2011); see Table 2.

Finally, we note that our adoption of $R=100$ as the radioloudness threshold, instead of the conventional (and more conservative) threshold of $R=10 \quad$ (e.g., Kellermann et al. 1989), is intended to exclude only sources that are more representative of the RLQ population (Ivezić et al. 2002), for which the potential effects of continuum boosting are expected to be more pronounced. Our HIZ, BL04, and WLQ samples include four sources ([HB89] 0329-385, UM 645, SDSS J1733+5400, and SDSS J2102+0020), three sources (PG 1211+143, PG 1309+355, and PG 1425+267), and one source (SDSS J1141+0219), respectively, with $10<R<100$.
Table 3

Spearman-rank Correlation Coefficients

\begin{tabular}{|c|c|c|c|c|}
\hline Correlation & Sample & $N$ & $r_{\mathrm{S}}$ & $p$ \\
\hline$\overline{\mathrm{EW}}(\mathrm{C}$ IV $)-\nu L_{\nu}(5100 \AA)$ & BL04 & 63 & -0.23 & $7.37 \times 10^{-2}$ \\
\hline $\mathrm{EW}(\mathrm{C}$ Iv $)-L / L_{\text {Edd }}$ & BL04 & 63 & -0.56 & $2.11 \times 10^{-6}$ \\
\hline $\mathrm{EW}(\mathrm{C}$ IV $)-\nu L_{\nu}(5100 \AA)$ & HIZ & 36 & -0.38 & $2.32 \times 10^{-2}$ \\
\hline $\mathrm{EW}(\mathrm{C}$ Iv $)-L / L_{\mathrm{Edd}}$ & HIZ & 36 & -0.30 & $7.74 \times 10^{-2}$ \\
\hline $\mathrm{EW}(\mathrm{C}$ IV $)-\nu L_{\nu}(5100 \AA)$ & BL04 and HIZ & 99 & -0.33 & $8.35 \times 10^{-4}$ \\
\hline $\mathrm{EW}(\mathrm{C}$ IV $)-L / L_{\mathrm{Edd}}$ & BL04 and HIZ & 99 & -0.52 & $3.03 \times 10^{-8}$ \\
\hline
\end{tabular}

Note. The last three columns represent the number of sources in each correlation, the Spearman-rank correlation coefficient, and the chance probability, respectively.

\section{RESULTS}

We plot EW(C IV $)$ versus $\nu L_{\nu}(5100 \AA)$ and $L / L_{\text {Edd }}$ for the BL04 and HIZ samples in Figure 1, and present the respective Spearman-rank correlation coefficients $\left(r_{\mathrm{S}}\right)$ and chance probabilities $(p)$ in Table 3. Our results for the BL04 sample 
indicate that $\mathrm{EW}(\mathrm{C}$ IV $)$ and $L / L_{\mathrm{Edd}}$ are significantly anticorrelated (i.e., $p<1 \%$ ), whereas no significant correlation is observed between $\mathrm{EW}(\mathrm{C}$ IV $)$ and $\nu L_{\nu}(5100 \AA)$, consistent with the BL04 finding. For the HIZ sample, there is no significant correlation between $\mathrm{EW}(\mathrm{CIV})$ and either $L / L_{\mathrm{Edd}}$ or $\nu L_{\nu}$ $(5100 \AA)$. The lack of an $\mathrm{EW}(\mathrm{C}$ IV $)-\nu L_{\nu}(5100 \AA)$ anti-correlation in our HIZ sample, as might have been expected from the classical Baldwin effect, may be due to obtaining $\mathrm{C}$ IV and $\nu L_{\nu}(5100 \AA)$ from two different and non-contemporaneous spectra for each source, as well as including different sources for $\mathrm{C}$ IV data with different measurement techniques (see Table 1). We do find, however, a significant anticorrelation between $\mathrm{EW}(\mathrm{C}$ IV $)$ and $\nu L_{\nu}(1450 \AA)$ for the HIZ sources, consistent with the Baldwin (1977) result. When the BL04 and HIZ samples are combined, both $\nu L_{\nu}(5100 \AA)$ and $L / L_{\text {Edd }}$ are significantly anti-correlated with $\mathrm{EW}(\mathrm{C}$ IV $)$, although the anti-correlation with $L / L_{\mathrm{Edd}}$ is substantially stronger and it is stronger than the $\operatorname{EW}(\mathrm{C}$ IV $)-L / L_{\mathrm{Edd}}$ anticorrelation for the BL04 sample alone ( $p$ drops from $2.11 \times 10^{-6}$ to $\left.3.03 \times 10^{-8}\right)$, thus bolstering the BL04 results. We also note that, when replacing either $\nu L_{\nu}(5100 \AA)$ or $L / L_{\text {Edd }}$ by source redshift, the above correlations with $\mathrm{EW}\left(\mathrm{C}_{\mathrm{IV}}\right)$ weaken considerably. These results indicate that the MBE is more pronounced at lower luminosities and thus lower redshifts, where several low-luminosity sources with relatively high Eddington ratios are observed (Figure 1). At high redshift, it is difficult to obtain high-quality spectral information for low-luminosity quasars. This practical limitation results in a strong dependence between $L$ and $L / L_{\text {Edd }}$, and thus relatively high- $L$ sources have narrow ranges of both $L$ and $L / L_{\text {Edd }}$, which may also explain why we do not detect a significant $\mathrm{EW}(\mathrm{C}$ IV $)-L / L_{\mathrm{Edd}}$ anti-correlation for the HIZ sample alone. In fact, the BL04 sample spans the $0.01 \lesssim L / L_{\text {Edd }} \lesssim 1$ range, while the HIZ sample spans only the $0.1 \lesssim L / L$ Edd $\lesssim 1$ range.

Figure 1 also shows that WLQs, not included in any of the correlations, appear as outliers in these relations. To quantify the deviation of WLQs from the $\mathrm{EW}(\mathrm{C}$ IV $)-L / L_{\mathrm{Edd}}$ anti-correlation, we fitted a linear model to the $\log [\mathrm{EW}(\mathrm{C}$ IV $)]$ and $\log \left(L / L_{\mathrm{Edd}}\right)$ values of sources from the combined BL04 and HIZ samples. A standard $\chi^{2}$ minimization, weighted by the errors on $\log \left[\mathrm{EW}\left(\mathrm{C}_{\mathrm{IV}}\right)\right]$, yielded an unsatisfactory fit (with $\left.\chi^{2} / \nu=5191 / 97\right)$, indicating that either a linear model does not provide the best fit, the error bars on $\log \left[\mathrm{EW}\left(\mathrm{C}_{\mathrm{IV}}\right)\right]$ are underestimated, or that additional scatter in the data must be taken into account. Assuming a linear model with $\chi^{2} / \nu=97 / 97$, we find an additional scatter in the $\log [\mathrm{EW}(\mathrm{C}$ Iv $)]$ values of $\sim 0.2$ dex (see, e.g., Tremaine et al. 2002; Kaspi et al. 2005); this scatter is much larger than the typical measurement errors on $\log \left[\mathrm{EW}\left(\mathrm{C}_{\mathrm{IV}}\right)\right]$. One likely source for this scatter stems from the fact that the $\mathrm{C}$ IV and $\mathrm{H} \beta$ spectral information are obtained from different datasets and are non-contemporaneous. But as we discuss in Section 4, additional physical parameters may also contribute to this scatter. We account for this potential intrinsic scatter by using the bivariate correlated errors and scatter method (BCES; Akritas \& Bershady 1996) for performing the linear regression. Since a derivation of the Eddington ratio involves a typical uncertainty of $\sim 0.3$ dex, we assign to all the $\log \left(L / L_{\mathrm{Edd}}\right)$ values of the BL04 and HIZ samples homoscedastic errors of 0.3 dex (cf. Section 3.2.2 of Shemmer et al. 2008). The BCES

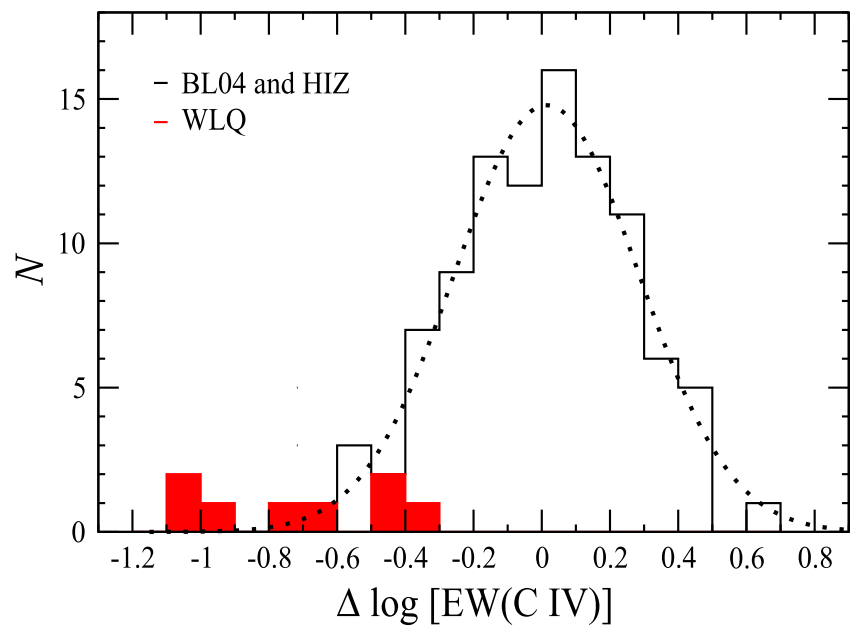

Figure 2. Distribution of $\log [\mathrm{EW}(\mathrm{C}$ Iv $)]$ residuals, computed as the difference between observed $\log [\mathrm{EW}(\mathrm{C}$ IV $)]$ values and $\log [\mathrm{EW}(\mathrm{C}$ IV $)]$ values predicted from the BCES Bisector best-fit $\log [\mathrm{EW}(\mathrm{C}$ IV $)]-\log \left(L / L_{\text {Edd }}\right)$ relation. Sources from the BL04 and HIZ samples are represented by the unshaded histogram; the dotted curve is the best-fit Gaussian distribution for this histogram with $\sigma=0.27$ dex. Five of the nine WLQs (shaded histogram) lie below the $\sim 3 \sigma$ threshold of the best-fit Gaussian distribution (including the WLQ SDSS J114153.34+021924.3 with $\Delta \log \left[\mathrm{EW}\left(\mathrm{C}_{\mathrm{IV}}\right)\right] \simeq-2.0$ which is not included in the shaded histogram, for clarity).

Bisector best-fit relation for the BL04 and HIZ samples,

$\log [\mathrm{EW}(\mathrm{C}$ IV $)]=(-0.58 \pm 0.07) \log \left(L / L_{\mathrm{Edd}}\right)+(1.35 \pm 0.04)$

is plotted in the right panel of Figure 1. We cross-checked the above BCES Bisector relation against the results from a linearregression analysis using the maximum-likelihood estimate method of Kelly (2007). This method results in a flatter slope $(-0.41 \pm 0.08)$ and a roughly similar intercept $(1.44 \pm 0.04)$, but the slope is highly sensitive to the uncertainties assumed on $\log \left(L / L_{\text {Edd }}\right)$ in the sense that the best-fit relation steepens as the errors increase beyond $0.3 \mathrm{dex}$. We adopt the more conservative BCES Bisector relation since, as shown below, this provides more stringent constraints on the WLQ sample.

In Figure 2 we plot a distribution of the differences between the observed $\log \left[\mathrm{EW}\left(\mathrm{C}_{\mathrm{IV}}\right)\right]$ values and those predicted from the $L / L_{\text {Edd }}$ values of the sources, based on Equation (2). The distribution of these residuals, $\Delta \log \left[\mathrm{EW}\left(\mathrm{C}_{\mathrm{IV}}\right)\right]$, for the BL04 and HIZ samples is roughly symmetric with zero mean and extreme values of \pm 0.6 dex. The best-fit Gaussian model to this distribution gives $\mu=-0.04 \mathrm{dex}$ and $\sigma=0.27 \mathrm{dex}$. All sources from the WLQ sample lie at $\gtrsim 1.5 \sigma$ below the mean of this Gaussian distribution and five of these lie at $\gtrsim 3 \sigma$ (Figure 2). The three WLQs with the largest (less negative) residuals $(-0.46 \leqslant \Delta \log [\mathrm{EW}(\mathrm{C}$ Iv $)] \leqslant-0.39$ dex $)$, PHL 1811, SDSS J1521+5202, and SDSS J1447-0203, overlap with the residuals of the combined BL04 and HIZ samples (although SDSS J1521 +5202 will lie below the $3 \sigma$ threshold if we adopt the Shen et al. 2011 EW measurement); we discuss these sources further in Section 4.

\section{DISCUSSION}

In this work, we expanded the BL04 parameter space by including sources having the highest possible redshifts and luminosities, for which high-quality spectral information for 
$\mathrm{C}$ IV and $\mathrm{H} \beta$ is available, in order to test whether the relative strength of $\mathrm{C}$ IV depends primarily on $L / L_{\mathrm{Edd}}$. We find that, for ordinary quasars across the $10^{43} \lesssim \nu L_{\nu}(5100 \AA) \lesssim 10^{47} \mathrm{erg} \mathrm{s}^{-1}$ and $0.01 \lesssim L / L_{\text {Edd }} \lesssim 1$ ranges, the scatter in the Baldwin effect is minimized when $L / L_{\text {Edd }}$ replaces monochromatic luminosity, thus extending the $\mathrm{EW}(\mathrm{C}$ IV $)-L / L_{\text {Edd }}$ anti-correlation from BL04, i.e., the MBE, by almost two orders of magnitude in luminosity. However, we also find no significant correlations between $\mathrm{EW}\left(\mathrm{C}_{\mathrm{IV}}\right)$ and either $\nu L_{\nu}(5100 \AA)$ or $L / L_{\text {Edd }}$ when only high-redshift and high-luminosity sources are considered; this is mainly a consequence of additional scatter introduced by using diverse data sets and the strong dependence between $\nu L_{\nu}(5100 \AA)$ and $L / L_{\text {Edd }}$ at high redshift. We also investigate how WLQs fit into this picture and whether they have exceptionally high Eddington ratios. We find that, in general, the $\mathrm{H} \beta$-based Eddington ratios of WLQs are within the norm when compared to ordinary quasars with similar redshifts and luminosities (see, e.g., Tables 1 and 2), and that most WLQs deviate considerably from the $\mathrm{EW}(\mathrm{C}$ IV $)-L / L_{\text {Edd }}$ anti-correlation, suggesting that the MBE may not be applicable to all quasars. If the strong deviation of these WLQs is due to selection effects, then low- $L / L_{\text {Edd }}$ sources with $\mathrm{EW}\left(\mathrm{C}_{\mathrm{IV}}\right) \approx 10^{2}-10^{3} \AA$ are required in order to cause the necessary steepening in the MBE for accommodating additional WLQs. It will be interesting to see whether the emerging population of high-EW(C IV $)$ quasars at high redshift would produce such an effect (e.g., Ross et al. 2014).

The fact that most WLQs do not follow the MBE may, instead, bring into question the reliability of determining $M_{\mathrm{BH}}$ values in WLQs and perhaps in ordinary quasars as well. Our linear regression analysis already indicates a trend of a steeper best-fit $\mathrm{EW}(\mathrm{C}$ IV $)-L / L_{\text {Edd }}$ relation as the assumed uncertainties on $L / L_{\text {Edd }}$ increase. Such a steepening may accommodate some, but perhaps not all, WLQs in the MBE. The standard, single-epoch $\mathrm{H} \beta$ method for obtaining $M_{\mathrm{BH}}$ and $L / L_{\mathrm{Edd}}$, briefly outlined in Section 1 and given in Equation (1), respectively, is likely too simplistic, and may involve uncertainties much larger than 0.3 dex (as we assume in Section 3 ) as well as systematic uncertainties. One such systematic uncertainty may be a consequence of orientation bias (see, e.g., Shen \& Ho 2014). In this scenario, sources viewed close to pole-on exhibit narrower BELR lines, and thus their actual $M_{\mathrm{BH}}\left(L / L_{\mathrm{Edd}}\right)$ values should be higher (lower). If WLQs suffer from orientation bias, then their Eddington ratios should be even smaller than those in Table 2, resulting in a larger deviation from the MBE. Orientation bias is, therefore, an unlikely explanation for this deviation. A different method of determining Eddington ratios in WLQs is required to test whether these ratios are considerably larger than the respective $\mathrm{H} \beta$-based values. The hard-X-ray photon index $(\Gamma)$ is one such $L / L_{\text {Edd }}$ indicator that can be used for cross-checking with $\mathrm{H} \beta$ based values (e.g., Shemmer et al. 2008). To this end, such a comparison has been made for two WLQs, PHL 1811 and SDSS J1141+0219; for both sources the X-ray-based $L / L_{\mathrm{Edd}}$ value is consistent with the $\mathrm{H} \beta$-based value (see Leighly et al. 2007b and Shemmer et al. 2010, respectively). Based on Equation (2),WLQs are expected to have $L / L_{\text {Edd }} \gtrsim 4$, which would render extremely steep hard-X-ray spectra with $\Gamma \gtrsim 3$ (Shemmer et al. 2008). X-ray spectroscopy of a statistically meaningful sample of WLQs may therefore provide a robust test of the hypothesis that WLQs are sources with extremely high Eddington ratios.
Alternatively, WLQs may be pointing to the fact that additional physical properties may play a role in determining the relative strength of the $\mathrm{C}$ IV line. From a chronological perspective, the classical Baldwin effect (Baldwin 1977), observed for high-redshift quasars (for practical reasons), included substantial scatter which, as we explain in Section 3, could not have been effectively minimized by replacing the luminosity with the Eddington ratio. Mainly low-luminosity sources with high Eddington ratios, e.g., narrow-line Seyfert 1 galaxies (NLS1s), led BL04 to conclude that $L / L_{\text {Edd }}$ is the primary physical parameter governing the relative strength of C IV. NLS1s deviate considerably from the classical Baldwin effect, but are accommodated by the MBE. In this work, we show that most of our WLQs deviate considerably from the MBE (and from the classical Baldwin effect), revealing that the relative strength of C IV may not depend solely on $L / L_{\mathrm{Edd}}$ for all quasars. In this respect, WLQs are analogous to NLS1s by calling for more scrutiny into the parameters controlling BELR line strengths in quasars.

BL04 explored additional observables that may further reduce the scatter in the MBE. For example, they found that the combination of $L / L_{\mathrm{Edd}}$ and the $\mathrm{EW}$ of the $[\mathrm{O}$ III] $\lambda 5007$ narrow emission line provided the strongest anti-correlation with EW(C IV). Only one of our WLQs, SDSS J1447-0203, has [O III] emission lines tentatively detected; the spectra of the other four WLQs from Plotkin et al. (2015) do not cover the [O III] lines. Our other four WLQs, SDSS J1141+0219, SDSS J1237+6301, SDSS J1521+5202, and PHL 1811 (as well as about a quarter of the sources from the HIZ sample; see Netzer et al. 2004; N07) have tight upper limits on EW([O III]). While this may be consistent with the general trend of weaker $\mathrm{C}$ IV lines in sources with weaker [O III] emission (see BL04 and references therein), the limited $\left[\mathrm{O}_{\mathrm{III}}\right]$ statistics prevent us from testing whether this observable can explain part or all of the WLQ deviation. We note, however, that the relative strength of [O III] as well as other observables studied by BL04 may all be governed primarily by the Eddington ratio.

Additional parameters that may affect the relative strength of the $\mathrm{C}$ IV line can be split broadly into properties of the i) SED, and ii) BELR. A high Eddington ratio results in a softer, UVpeaked SED, and this may naturally explain relatively weak C IV lines due to the paucity of highly ionizing photons; this model has been suggested for explaining the unusual properties of PHL 1811 (e.g., Leighly et al. 2007a). It is interesting to note that PHL 1811, its high-redshift "analog", SDSS J1521+5202 (Wu et al. 2011), as well as SDSS J1447-0203 which Plotkin et al. (2015) consider a "borderline" WLQ (or, an extreme "wind-dominated" quasar), appear to follow the MBE (within $\sim 1.5 \sigma-2 \sigma$; see Section 3 ). These sources may be different than the rest of the WLQs in our sample in the sense that the Eddington ratio alone may explain their weak $\mathrm{C}$ IV lines. ${ }^{4}$ Alternatively, the difference between PHL 1811-like sources and the other, more extreme WLQs may be related to SED shielding (or modification) and orientation effects (Wu et al. 2011). We emphasize that the $\mathrm{EW}(\mathrm{C}$ IV $)<10 \AA$ criterion we adopt for

\footnotetext{
4 Although this does not necessarily imply that these three sources belong to a single quasar subclass. In particular, they differ in their X-ray properties; SDSS J1447-0203 and SDSS J1521+5202 exhibit an effective power-law photon index $(\Gamma)$ of $>1.0$ and $0.6 \pm 0.2$, respectively, in the observed-frame $0.5-8 \mathrm{keV}$ band, indicating significant intrinsic absorption at least in the latter source (Luo et al. 2015), and PHL 1811 exhibits $\Gamma=2.3 \pm 0.1$ in the observed-frame $0.3-5 \mathrm{keV}$ band with no detectable intrinsic absorption (Leighly et al. 2007b).
} 
WLQs is statistically driven and it depends on the particular quasar sample under consideration (see Section 2). It is more instructive, perhaps, to use a physically motivated definition for WLQs as being clear outliers from the MBE, deviating by more than $3 \sigma$ from this relation on the low-EW end, i.e., sources having $\Delta \log \left[\mathrm{EW}\left(\mathrm{C}_{\mathrm{IV}}\right)\right] \lesssim-0.8$, based on this work. Given this definition, only five sources in our sample (i.e., further excluding SDSS J1237+6301 with $\Delta \log [\mathrm{EW}(\mathrm{C}$ IV $)] \sim-0.7)$ can be considered as WLQs, i.e., sources for which the $\mathrm{H} \beta$ based $L / L_{\text {Edd }}$ value may not fully explain their $\mathrm{C}$ IV line weakness. Finally, we note that a "cold" accretion disk, due to high $M_{\mathrm{BH}}$ values, has also been offered to explain the weak $\mathrm{C}$ IV lines in WLQs (Laor \& Davis 2011); detailed UV spectroscopy of WLQs is required to test the predictions of this model.

A variety of BELR physical properties can also affect the relative strength of the CIV line, such as the BELR geometry, covering factor, density, and metallicity. Extremely weak C IV lines, such as those observed in WLQs with $\Delta \log \left[\mathrm{EW}\left(\mathrm{C}_{\mathrm{IV}}\right)\right] \lesssim-0.8$, may be attributed to a deficiency of gas in the BELR (i.e., an "anemic" BELR; Shemmer et al. 2010), or to an early evolutionary stage in the quasar's duty cycle where the BELR just started to form (e.g., Hryniewicz et al. 2010). A more rigorous investigation of the parameters controlling the relative strengths of BELR lines in quasars, which is beyond the scope of this work, should include a comprehensive analysis of spectral information for low- and high-ionization BELR lines as well as the SED shape, in conjunction with photoionization modeling, for a quasar sample much larger than studied herein. Most importantly, the relative strengths of high-ionization BELR lines, such as $\mathrm{C}_{\text {IV }}$ with $\chi_{\text {ion }}=47.9 \mathrm{eV}$, should be investigated jointly with the relative strengths of low-ionization BELR lines, such as $\mathrm{H} \beta$ with $\chi_{\text {ion }}=13.6 \mathrm{eV}$ or $\mathrm{Mg}$ II with $\chi_{\text {ion }}=7.6 \mathrm{eV}$. For example, correlations involving ratios of the relative strengths of these lines, such as $\mathrm{EW}(\mathrm{C} \mathrm{IV}) / \mathrm{EW}(\mathrm{H} \beta)$, as well as the X-rayto-optical SED should be investigated in more detail (e.g., BL04; Wu et al. 2011, 2012; Plotkin et al. 2015). Furthermore, it is necessary to decompose the BELR lines into "disk" and "wind" (or outflow) components, in particular for C IV (e.g., Richards et al. 2011), in order to check whether the $\mathrm{EW}$ of each component of the line profile is correlated with a fundamental physical property, such as $L / L_{\text {Edd }}$. Detailed lineprofile measurements, yielding emission-line blueshifts and line asymmetries, should provide additional insights (e.g., Richards et al. 2011).

\section{CONCLUSIONS}

We utilize a sample of 99 ordinary quasars across wide ranges of luminosity and redshift to show that the relative strength of the broad $\mathrm{C}$ IV line is primarily anti-correlated with the $\mathrm{H} \beta$-based Eddington ratio, i.e., an MBE, thus confirming and extending previous work limited to nearby, low-luminosity sources. We also find that all nine WLQs with available $\mathrm{H} \beta$ and $\mathrm{C}_{\text {IV }}$ information in the archive have typical $\mathrm{H} \beta$-based $L / L_{\text {Edd }}$ values in contrast with the extremely high values expected from the MBE. While the EWs of the $C_{\text {IV }}$ lines in four of these WLQs are consistent with the MBE, the other five WLQs deviate significantly from this relation by exhibiting EWs much smaller than predicted from their $\mathrm{H} \beta$-based $L / L_{\text {Edd }}$ values. In case the single-epoch $\mathrm{H} \beta$ method can provide a reliable determination of $M_{\mathrm{BH}}$ in all quasars, then our results indicate that $\mathrm{EW}(\mathrm{C}$ IV $)$ cannot depend solely on $L / L_{\mathrm{Edd}}$. While a comprehensive investigation into the nature of the MBE is beyond the scope of this study, we outline additional spectroscopic work required to determine the roles that basic quasar physical properties play in controlling the relative strengths of broad-emission lines in quasars.

We thank Marcia Lieber and Richard Plotkin for fruitful discussions. We gratefully acknowledge a helpful and constructive report from an anonymous referee, who helped to improve this work. This research has made use of the NASA/IPAC Extragalactic Database (NED) which is operated by the Jet Propulsion Laboratory, California Institute of Technology, under contract with the National Aeronautics and Space Administration.

\section{REFERENCES}

Akritas, M. G., \& Bershady, M. A. 1996, ApJ, 470, 706

Bachev, R., Marziani, P., Sulentic, J. W., et al. 2004, ApJ, 617, 171

Baldwin, J. A. 1977, ApJ, 214, 679

Bañados, E., Venemans, B. P., Morganson, E., et al. 2014, AJ, 148, 14

Baskin, A., \& Laor, A. 2004, MNRAS, 350, L31

Baskin, A., \& Laor, A. 2005, MNRAS, 356, 1029

Becker, R. H., White, R. L., \& Helfand, D. J. 1995, ApJ, 450, 559

Bentz, M. C., Peterson, B. M., Netzer, H., Pogge, R. W., \& Vestergaard, M 2009, ApJ, 697, 160

Boroson, T. A., \& Green, R. F. 1992, ApJS, 80, 109

Brandt, W. N., Laor, A., \& Wills, B. J. 2000, ApJ, 528, 637

Brotherton, M. S., \& Francis, P. J. 1999, Quasars and Cosmology, 162, 395 Condon, J. J., Cotton, W. D., Greisen, E. W., et al. 1998, AJ, 115, 1693

Croom, S. M., Smith, R. J., Boyle, B. J., et al. 2004, MNRAS, 349, 1397

Diamond-Stanic, A. M., Fan, X., Brandt, W. N., et al. 2009, ApJ, 699, 782

Dietrich, M., Hamann, F., Shields, J. C., et al. 2002, ApJ, 581, 912

Dong, X.-B., Wang, T.-G., Wang, J.-G., et al. 2009, ApJL, 703, L1

Espey, B. R., Carswell, R. F., Bailey, J. A., Smith, M. G., \& Ward, M. J. 1989, ApJ, 342, 666

Fan, X., Strauss, M. A., Gunn, J. E., et al. 1999, ApJL, 526, L57

Fan, X., Strauss, M. A., Richards, G. T., et al. 2006, AJ, 131, 1203

Forster, K., Green, P. J., Aldcroft, T. L., et al. 2001, ApJS, 134, 35

Gibson, R. R., Jiang, L., Brandt, W. N., et al. 2009, ApJ, 692, 758

Green, P. J., Forster, K., \& Kuraszkiewicz, J. 2001, ApJ, 556, 727

Hryniewicz, K., Czerny, B., Nikołajuk, M., \& Kuraszkiewicz, J. 2010, MNRAS, 404, 2028

Ivezić, Ž., Menou, K., Knapp, G. R., et al. 2002, AJ, 124, 2364

Jannuzi, B. T., Bahcall, J. N., Bergeron, J., et al. 1998, ApJS, 118, 1

Just, D. W., Brandt, W. N., Shemmer, O., et al. 2007, ApJ, 665, 1004

Kaspi, S., Maoz, D., Netzer, H., et al. 2005, ApJ, 629, 61

Kellermann, K. I., Sramek, R., Schmidt, M., Shaffer, D. B., \& Green, R. 1989, AJ, 98, 1195

Kelly, B. C. 2007, ApJ, 665, 1489

Laor, A. 1998, ApJL, 505, L83

Laor, A., \& Davis, S. W. 2011, MNRAS, 417, 681

Leighly, K. M., Halpern, J. P., Jenkins, E. B., \& Casebeer, D. 2007a, ApJS, 173,1

Leighly, K. M., Halpern, J. P., Jenkins, E. B., et al. 2007b, ApJ, 663, 103

Luo, B., Brandt, W. N., Hall, P. B., et al. 2015, ApJ, 805, 122

Malkan, M. A., Green, R. F., \& Hutchings, J. B. 1987, ApJ, 322, 729

Marconi, A., Risaliti, G., Gilli, R., et al. 2004, MNRAS, 351, 169

Meusinger, H., \& Balafkan, N. 2014, A\&A, 568, AA114

Netzer, H., Lira, P., Trakhtenbrot, B., Shemmer, O., \& Cury, I. 2007, ApJ, 671,1256

Netzer, H., Shemmer, O., Maiolino, R., et al. 2004, ApJ, 614, 558

Osmer, P. S., \& Shields, J. C. 1999, Quasars and Cosmology, 162, 235

Osmer, P. S., \& Smith, M. G. 1977, ApJ, 213, 607

Plotkin, R. M., Anderson, S. F., Brandt, W. N., et al. 2010, AJ, 139, 390

Plotkin, R. M., Shemmer, O., Trakhtenbrot, B., et al. 2015, ApJ, 805, 123

Richards, G. T., Kruczek, N. E., Gallagher, S. C., et al. 2011, AJ, 141, 167

Ross, N. P., Hamann, F., Zakamska, N. L., et al. 2014, arXiv:1405.1047

Schneider, D. P., Schmidt, M., \& Gunn, J. E. 1991, AJ, 101, 2004

Shang, Z., Wills, B. J., Robinson, E. L., et al. 2003, ApJ, 586, 52

Shemmer, O., Brandt, W. N., Anderson, S. F., et al. 2009, ApJ, 696, 580

Shemmer, O., Brandt, W. N., Netzer, H., Maiolino, R., \& Kaspi, S. 2008, ApJ, 682,81 
Shemmer, O., Netzer, H., Maiolino, R., et al. 2004, ApJ, 614, 547

Shemmer, O., Trakhtenbrot, B., Anderson, S. F., et al. 2010, ApJL, 722, L152

Shen, Y., \& Ho, L. C. 2014, Natur, 513, 210

Shen, Y., \& Liu, X. 2012, ApJ, 753, 125

Shen, Y., Richards, G. T., Strauss, M. A., et al. 2011, ApJS, 194, 45

Spergel, D. N., Bean, R., Doré, O., et al. 2007, ApJS, 170, 377

Trakhtenbrot, B., \& Netzer, H. 2012, MNRAS, 427, 3081

Tremaine, S., Gebhardt, K., Bender, R., et al. 2002, ApJ, 574, 740

Trump, J. R., Hall, P. B., Reichard, T. A., et al. 2006, ApJS, 165, 1

Turnshek, D. A., \& Grillmair, C. J. 1986, ApJL, 310, L1

Vanden Berk, D. E., Richards, G. T., Bauer, A., et al. 2001, AJ, 122, 549
Wilkes, B. J., Kuraszkiewicz, J., Green, P. J., Mathur, S., \& McDowell, J. C. 1999, ApJ, 513, 76

Wills, B. J., Brotherton, M. S., Fang, D., Steidel, C. C., \& Sargent, W. L. W. 1993, ApJ, 415, 563

Wills, B. J., Laor, A., Brotherton, M. S., et al. 1999, ApJL, 515, L53

Wu, J., Brandt, W. N., Anderson, S. F., et al. 2012, ApJ, 747, 10

Wu, J., Brandt, W. N., Hall, P. B., et al. 2011, ApJ, 736, 28

Xu, Y., Bian, W.-H., Yuan, Q.-R., \& Huang, K.-L. 2008, MNRAS, 389, 1703

York, D. G., Adelman, J., Anderson, J. E., Jr, et al. 2000, AJ, 120, 1579

Zheng, W., \& Malkan, M. A. 1993, ApJ, 415, 517 